\title{
Brown Dwarf Kinematics Project (BDKP)
}

\author{
J. Faherty ${ }^{1}$, K. Cruz ${ }^{2}$, A. Burgasser ${ }^{3}$, F. Walter ${ }^{1}$ and M. Shara \\ ${ }^{1}$ Stony Brook University, Stony Brook, NY, 11794 email: jfaherty@amnh.org \\ ${ }^{2}$ Caltech, MC 105-24 Pasadena, CA 91125 email: kelle@astro.caltech.edu \\ ${ }^{3}$ MIT,77 Massachusetts Avenue Cambridge, MA 02139 email:ajb@mit.edu \\ ${ }^{4}$ American Museum of Natural History, 79th Street and CPW, New York, NY 10023 \\ email:mshara@amnh.org
}

\begin{abstract}
We report on the progress of the Brown Dwarf Kinematics Project (BDKP), which aims to measure the $6 \mathrm{D}$ positions and velocities of all known brown dwarfs within $20 \mathrm{pc}$ of the Sun and select sources of scientific interest. In this paper we report on the status of the 33 targets on our parallax list as well as the results of our proper motion survey where we have measured over 400 new proper motions for known late M, L and T dwarfs.
\end{abstract}

Keywords. stars: low-mass, brown dwarfs, stars: kinematics, stars: fundamental parameters

\section{Introduction}

Brown dwarfs are faint, substellar mass objects that share properties of both stars and planets. They encompass three spectral classes; late type M, L, and T with over 600 members in the lowest temperature spectral classes (L and T). Brown Dwarfs offer a link between planet and star formation, challenges for atmospheric models, and clues into the early history of our galaxy. Only a small percentage of all identified objects have full 6D positions: $13 \%$ of known $\mathrm{L}$ and $\mathrm{T}$ dwarfs have reported parallaxes, less than half have proper motion measurements, and a sparse $8 \%$ have measured radial velocities. Despite these limited statistics the kinematics of late-type dwarfs have already provided insight into the origins and long-term evolution of this low mass population. From the analysis of space velocities of a sample of $21 \mathrm{~L}$ and $\mathrm{T}$ dwarfs within $20 \mathrm{pc}$ of the Sun, Osorio, et al. (2007) have concluded that as a group these objects are relatively young (mean age $\sim 1$ Gyr), a finding inconsistent with population synthesis simulations (Burgasser 2004). Furthermore, nearly half of their sample appears to have kinematics consistent with membership in the Hyades moving group ( $625 \mathrm{Myr})$. This implies that many of the nearby brown dwarfs may be very low mass members of young recently uncovered moving groups (Zuckerman \& Song 2004). We currently have 33 confirmed brown dwarfs on a parallax list, most of which show potential membership in young associations. We also have a large observing program to measure proper motions for all known $\mathrm{L}$ and $\mathrm{T}$ dwarfs so we can begin a kinematic analysis of the entire population of substellar mass objects.

\section{Overview of parallax program}

We are using the ANDICAM $1024 \times 1024$ IR Hawaii detector on the $1.3 \mathrm{~m}$ telescope at CTIO to measure brown dwarf parallaxes in the $\mathrm{J}$ band. Our instrument has a plate scale of 0.137 " / pixel and a 2.4 arcminute field of view. Our target list (see table 1.) contains 3 L-subdwarfs, 11 late M to early L's that show gravity sensitive features indicative of youth,14 early to late L's that have photometric distances within 20pc and 4 Calibrators (not shown) with previously measured parallaxes. All of our parallax frames contain at 
Table 1. PARALLAX TARGETS

\begin{tabular}{rrrrccc||ccccccc}
\hline ID & P.M. & SpT. & $J$ & $H$ & \multicolumn{1}{c}{$K$} & \multicolumn{1}{c}{ N ID } & P.M. & SpT. & $J$ & $H$ & $K$ & N \\
\hline 6 & - & Lsd & 12.91 & 12.30 & 12.02 & 8 & 20 & 878 & L1.5 & 12.45 & 11.69 & 11.15 & 4 \\
7 & - & Lsd & 14.54 & 14.12 & 13.88 & 4 & 21 & 769 & L2 & 12.81 & 12.04 & 11.46 & 9 \\
8 & - & Lsd & 12.55 & 12.14 & 11.93 & 7 & 22 & 574 & L0 & 13.58 & 11.69 & 11.15 & 4 \\
9 & - & M6 & 15.10 & 14.22 & 13.45 & 5 & 23 & 487 & L1 & 13.65 & 13.03 & 12.57 & 4 \\
10 & - & M9.5 & 13.60 & 12.90 & 12.39 & 5 & 24 & 447 & L2 & 13.41 & 12.57 & 11.98 & 8 \\
11 & 3600 & M9 & 11.38 & 10.82 & 10.39 & 7 & 25 & 426 & L5 & 14.49 & 13.63 & 13.05 & 4 \\
12 & 210 & L3 & 14.98 & 13.71 & 12.96 & 5 & 26 & 375 & L5 & 14.48 & 13.34 & 12.60 & 6 \\
13 & 150 & L0 & 15.07 & 14.00 & 13.42 & 7 & 27 & 329 & L7 & 15.51 & 14.44 & 13.73 & 5 \\
14 & 89 & M9 & 12.69 & 12.00 & 11.50 & 7 & 28 & 236 & L2 & 13.52 & 12.63 & 12.05 & 5 \\
15 & 68 & M8.5 & 13.97 & 13.28 & 12.81 & 6 & 29 & 194 & L6.5 & 14.41 & 13.37 & 12.81 & 8 \\
16 & 49 & M8.5 & 13.03 & 12.36 & 11.89 & 5 & 30 & - & L1.5 & 13.12 & 12.27 & 11.75 & 4 \\
17 & 41 & M8 & 13.63 & 12.97 & 12.45 & 4 & 31 & - & L6 & 15.48 & 14.35 & 13.56 & 4 \\
18 & - & L0 & 14.78 & 13.86 & 13.27 & 5 & 32 & - & L6 & 14.55 & 13.53 & 13.01 & 4 \\
19 & - & M6 & 12.76 & 11.77 & 11.28 & 5 & 33 & - & L7 & 15.19 & 14.08 & 13.34 & 4 \\
\hline \multicolumn{7}{c}{ Table 1: Proper Motions(P. M.) are in milli-arcsec/year. N is the number of parallax frames. } &
\end{tabular}

least 7 astrometric reference stars. The target objects are carefully placed on the same $\mathrm{X}, \mathrm{Y}$ pixel position at each observation so as to minimize differential distortions and ensure the same reference stars in each frame. Our initial transformations demonstrate an accuracy of 7-10 mas or better. The majority of objects are expected to be within $20 \mathrm{pc}$ therefore we expect to measure distances with $20 \%$ accuracy or better. We started observing our targets in January 2007 and have attempted to obtain at least one parallax frame per target per month while it is observable. Targets must be observed at least once every six months near times of maximum parallactic shift to accurately measure a parallax. Our observations will continue through January 2009 and we plan on reporting our preliminary measurements after 2 years of collected data.

\section{Overview of proper motion program}

We are using telescopes in the southern and northern hemisphere to measure proper motions for all known $\mathrm{L}$ and $\mathrm{T}$ dwarfs. As stated less than half of all known $\mathrm{L}$ and $\mathrm{T}$ dwarfs have measured proper motions. This is mainly due to the youthfulness of IR technology and the lack of a second epoch to follow-up on the first epochs of 8-10 years ago. We are using CPAPIR, a near-IR camera on the $1.5 \mathrm{~m}$ at CTIO, TIFKAM a near-IR imager on the $1.3 \mathrm{~m}$ at KPNO, and the I band imager on the $0.9 \mathrm{~m}$ at CTIO to target known brown dwarfs. To date we have measured proper motions for over 400 known late-type M,L, and T dwarfs. We calibrate our second epoch to the 2MASS catalogue and with an average baseline of 7.3 years obtain errors between 10 to 30 mas/yr for our targets. In our sample, $25 \%$ are moving faster than $0.5 \%$ /year, and $5 \%$ are moving faster then 1.0" / year. We are currently analyzing our sample for kinematic trends and will have a detailed paper out soon.

\section{References}

Burgasser, A. J. 2004, ApJS, 155, 191-207

Osorio, M. R. Z., Martin, E. L., Bejar, V. J. S., Bouy, H., Deshpande, R., \& Wainscoat, R. J. 2007, ApJ, 666, 1205-1218

Zuckerman, B. \& Song, I.. 2004, A\&AR, 42, 685-721 